International Journal of Pure and Applied Mathematics

Volume 84 No. 3 2013, 193-200

ISSN: 1311-8080 (printed version); ISSN: 1314-3395 (on-line version)

url: http://www.ijpam.eu

doi: http://dx.doi.org/10.12732/ijpam.v84i3.6

\title{
AN EXISTENCE AND UNIQUENESS THEOREM FOR AN $n$-TH ORDER FUNCTIONAL DIFFERENTIAL EQUATION
}

Fozi M. Dannan ${ }^{1}$, Elias G. Saleeby ${ }^{2} \S$

${ }^{1}$ Department of Basic Sciences

Arab International University

P.O. Box 10409, Damascus, SYRIA

${ }^{2}$ Department of Mathematics

Alfaisal University

P.O. Box 50927, Riyadh 11533, KINGDOM OF SAUDI ARABIA

Abstract: In this note we give a theorem for global existence and uniqueness of solutions for the initial value problem of an $n$-th order functional differential equation described by (1).

AMS Subject Classification: 34K05, 35G99

Key Words: functional differential equations, existence and uniqueness

\section{Introduction}

The purpose of this note is to study the existence and uniqueness of solutions of the initial value problem

$$
\begin{gathered}
f^{(n)}(x)+\sum_{i=1}^{n-1} a_{n-i}(x) f^{(n-i)}(x)=a(x) f(g(x))+b(x) f(x)+c(x), \\
f(0)=f_{0}, f^{\prime}(0)=f_{1}, \cdots, f^{(n-1)}(0)=f_{n-1},
\end{gathered}
$$

where $a(x) \neq 0, b(x), c(x)$ and $g(x)$ are continuous functions on $I:=[0, L]$, and $g(I) \subset I$, and $a_{i}(x)(i=1, \cdots, n-1)$ are at most differentiable functions on $I$, $L$ is a positive constant. Functional differential equation can be thought of as

Received: November 6, 2012

(C) 2013 Academic Publications, Ltd.

${ }^{\S}$ Correspondence author

$$
\text { url: www.acadpubl.eu }
$$


generalizations of ordinary differential equations, and so they appear as models in a good number of different settings - for example, in the study of wave motion, cell growth, wavelets etc. A large number of investigations of the solutions of equations that are special cases of (1), in both real and complex variables, have appeared (see for example, references [1] to [8], and references therein). Studies of equations of a more general type than (1) have also appeared (see for example [9],[10],[11]). However, the majority of these studies deal with equations of first or second order, and in fact, most of the earlier studies deal only with the existence and uniqueness of local solutions, and the characterization of solutions for the particular case when $g(x)=a x, a$ constant (not necessaily $<1$ ). In [10], Oberg notes that the question of local existence is meaningful only around a fixed point of $g$. In [8], global complex analytic solutions of (1) for $x$ a complex variable, under certain growth conditions on the coefficients, were characterized; and the existence and uniqueness of global solutions, when $x$ is real and $n=1$, were also established. The results in this note extend the analysis in [8] for $x$ real and $n>1$. The method of proof employed in [8] is more cumbersome to extend to the case $n>1$ than the method we develop in this article.

Let $X^{n}:=C^{n}(I, \mathbf{R})$, denotes the set of $n$-times differentiable functions on the compact subset $I \subset \mathbf{R}$. Let $E:=C(I, \mathbf{R})$. It is well known that $E$ is a Banach space with respect to the norm

$$
\|f\|=\sup _{x}\left(e^{-\gamma x}|f(x)|\right)
$$

for some $\gamma>0$, and that this norm is equivalent to the sup-norm $\|f\|_{\infty}:=$ $\sup _{0 \leq x \leq L}|f(x)|$ on $E$ (see [3],[12]). In our proof, we employ the following variant of the Schauder fixed point theorem (see [12], p.33).

Theorem (Schauder) Let $E$ be a Banach space, $G \subset E$ a nonempty convex bounded closed set and let $S: G \rightarrow G$ be a compact operator. Then $S$ has at least one fixed point.

\section{Existence and Uniqueness}

We now state and proof our theorem.

Theorem 1. Suppose that $a_{i}(i=1, \cdots, n-1), g, a \neq 0, b$ and $c$ belong to $E$, and that $g(I) \subset I$. Then, for any given constants $f_{i}, i=0,1, \cdots, n-1$,

(i) if also $a_{i} \in X^{i}, i=1, \cdots, n-1$, then (1) has at least one solution $f$ in $X^{n}$; 
(ii) (1) has a unique solution in $X$ provided that $g(x) \leq x$ on $I$.

Proof. First we prove (ii). Suppose (1) has two solutions $u(x)$ and $v(x)$ on $I$. Let $w(x):=u(x)-v(x)$. Then $w(x)$ satisfies

$$
\begin{aligned}
w^{(n)}(x)+\sum_{i=1}^{n-1} a_{n-i}(x) w^{(n-i)}(x) & =a(x) w(g(x))+b(x) w(x), \\
w(0) & =0, w^{\prime}(0)=0, \cdots, w^{(n-1)}(0)=0 .
\end{aligned}
$$

Now we convert (1) into an integral equation. The realtionship between linear differential equations and Volterra integral equations is given by Tricomi (see [13], p.18). Let $w^{(n)}(x)=z(x)$. Integrate from 0 to $x$, we obtain

$$
w^{(n-1)}(x)=\int_{0}^{x} z(s) d s .
$$

It is not difficult to see that by successive integrations along with interchanges in the order of integration yield

$$
w^{(n-i)}(x)=\int_{0}^{x} \frac{(x-s)^{i-1}}{(i-1) !} z(s) d s, i=1, \cdots, n .
$$

Using (2) to rewrite (1), we obtain

$$
\begin{aligned}
z(x)=a(x) & \int_{0}^{g(x)} \frac{(g(x)-s)^{n-1}}{(n-1) !} z(s) d s \\
& +\int_{0}^{x}\left(b(x) \frac{(x-s)^{n-1}}{(n-1) !}-\sum_{i=1}^{n-1} a_{n-i}(x) \frac{(x-s)^{i-1}}{(i-1) !}\right) z(s) d s .
\end{aligned}
$$

Since $g(x) \leq x$, we have that

$$
\begin{aligned}
& |z(x)| \leq \\
& \int_{0}^{x}\left(\frac{(x-s)^{n-1}}{(n-1) !}(|a(x)|+|b(x)|)+\sum_{i=1}^{n-1}\left|a_{n-i}(x)\right| \frac{(x-s)^{i-1}}{(i-1) !}\right)|z(s)| d s .
\end{aligned}
$$

Now, by the continuity of $a(x), b(x)$, and $a_{i}(x), i=1,2, \cdots, n-1$, we can rewrite (4) as

$$
|z(x)| \leq C \int_{0}^{x} \sum_{i=1}^{n-1} \frac{(x-s)^{i-1}}{(i-1) !}|z(s)| d s \leq C \int_{0}^{x} e^{(x-s)}|z(s)| d s,
$$


where $C:=\max _{0 \leq x \leq L}\left(|a(x)+b(x)|,\left|a_{1}(x)\right|, \cdots,\left|a_{i-1}(x)\right|\right)$. Moreover, let $U(s)=e^{-s}|z(s)|$. Then we have that

$$
U(x) \leq C \int_{0}^{x} U(s) d s .
$$

Putting $V(x)=\int_{0}^{x} U(s) d s$, we obtain from (6) that $V^{\prime}(x) \leq C V(x)$. Thus, $e^{-C x} V^{\prime}(x)-C V(x) e^{-C x}=\left(e^{-C x} V(x)\right)^{\prime} \leq 0$. Then by integration we get that $V(x) \leq 0$ and $U(x) \leq 0$. But $U(x) \geq 0$. Therefore, $U(x) \equiv 0$, and hence $z(x) \equiv 0$. Consequently, $w^{(n)}(x) \equiv 0$, and by the initial values we have that $w^{(n-1)}(x) \equiv \ldots \equiv w(x) \equiv 0$. This completes the proof of (ii).

Now we give the proof of (i). For convenience, and without loss of generality, we give the analysis for the $n=3$ case. Consider the equation

$$
f^{\prime \prime \prime}(x)+a_{2}(x) f^{\prime \prime}(x)+a_{1}(x) f^{\prime}(x)=H(x),
$$

where $H(x)=a(x) f(g(x))+b(x) f(x)+c(x)$. Integrate from 0 to $x$ three times, and use integration by parts, we obtain

$$
\begin{aligned}
f(x)= & f(0)+f^{\prime}(0) x+f^{\prime \prime}(0) \frac{x^{2}}{2}+a_{2}(0)\left(f(0) x+f^{\prime}(0) \frac{x^{2}}{2}\right) \\
& +\left(a_{1}(0)-a_{2}^{\prime}(0)\right) f(0) \frac{x^{2}}{2} \\
& -\int_{0}^{x}\left[a_{2}(s)-2(x-s) a_{2}^{\prime}(s)+\frac{(x-s)^{2}}{2} a_{2}^{\prime \prime}(s)\right] f(s) d s \\
& -\int_{0}^{x}\left[(x-s) a_{1}(s)-\frac{(x-s)^{2}}{2} a_{1}^{\prime}(s)\right] f(s) d s \\
& +\int_{0}^{x} \frac{(x-s)^{2}}{2} H(s) d s .
\end{aligned}
$$

Call this equation (F). Let $B$ be a bounded subset of $E$. For any $h \in B$, denote $f=S(h)$. From the expression of $f^{\prime}(x)$, under the continuity and differentiability assumptions on the $a_{i}$ 's, and the continuity assumptions on $a, b, c, g$ on $I$, we see that there is a constant $M>0$ such that $\left\|f^{\prime}\right\|_{\infty}<M$ for any $h$ in $B$. Now by the mean-value theorem, we have $|f(y)-f(x)| \leq M|y-x|$ for all $x, y \in I$. Taking $y=0$, we see that $|f(x)| \leq M L+|f(0)|$ for all $x$ in $I$. Then, by the Arzela-Ascoli theorem, the set $\{f=S(h) \mid h \in B\}$ has a convergent subsequence and hence $S: E \rightarrow E$ is a compact operator. 
Next, consider a closed ball $G$ of the space $E$ with norm $\|\cdot\|$, centered at zero and has radius $M$ - that is, $G:=\{f \in E:\|f\| \leq M\}$. Then for any given number $\gamma>0$, we obtain from $(\mathrm{F})$ that

$$
\begin{aligned}
|(S f)(x)| \leq & |f(0)|+\left|f^{\prime}(0)\right| L+\left|f^{\prime \prime}(0)\right| \frac{L^{2}}{2} \\
& +a_{2}|f(0)| L+a_{2}\left|f^{\prime}(0)\right| \frac{L^{2}}{2}+\left(a_{1}+a_{2}^{\prime}\right)|f(0)| \frac{L^{2}}{2} \\
& +\left(A_{2}+2 A_{2}^{\prime} L+A_{2}^{\prime \prime} \frac{L^{2}}{2}\right) \int_{0}^{x}|f(s)| e^{-\gamma s} e^{\gamma s} d s \\
& +\left(A_{1} L+A_{1}^{\prime} \frac{L^{2}}{2}\right) \int_{0}^{x}|f(s)| e^{-\gamma s} e^{\gamma s} d s \\
& +(A+B) \int_{0}^{x}|f(s)| e^{-\gamma s} e^{\gamma s} d s+C
\end{aligned}
$$

where $a_{i}=\left|a_{i}(0)\right|, a_{i}^{\prime}=\left|a_{i}^{\prime}(0)\right|, A_{i}=\max _{0 \leq x \leq L}\left|A_{i}(x)\right|, A_{i}^{\prime}=\max _{0 \leq x \leq L}\left|a_{i}^{\prime}(x)\right|$, $A_{i}^{\prime \prime}=\max _{0 \leq x \leq L}\left|a_{i}^{\prime \prime}(x)\right|, A=\max _{0 \leq x \leq L}|a(x)|, B=\max _{0 \leq x \leq L}|b(x)|$, and $C=\max _{0 \leq x \leq L}|c(x)|$. This last equation reduces to

$$
\begin{aligned}
|(S f)(x)| \leq & |f(0)|+\left|f^{\prime}(0)\right| L+\left|f^{\prime \prime}(0)\right| \frac{L^{2}}{2} \\
& +a_{2}|f(0)| L+a_{2}\left|f^{\prime}(0)\right| \frac{L^{2}}{2}+\left(a_{1}+a_{2}^{\prime}\right)|f(0)| \frac{L^{2}}{2} \\
& +\left(A_{2}+2 A_{2}^{\prime} L+A_{2}^{\prime \prime} \frac{L^{2}}{2}\right) M\left(\frac{e^{\gamma x}-1}{\gamma}\right) \\
& +\left(A_{1} L+A_{1}^{\prime} \frac{L^{2}}{2}\right) M\left(\frac{e^{\gamma x}-1}{\gamma}\right) \\
& +(A+B) M\left(\frac{e^{\gamma x}-1}{\gamma}\right)+C
\end{aligned}
$$

which in turn can be put into the short form

$$
|(S f)(x)|=K_{1}+K_{2} M \frac{e^{\gamma x}}{\gamma},
$$

where $K_{1}, K_{2}$ are appropriate constants. Then after multiplying the above inequality by $e^{-\gamma x}$,we obtain

$$
\|S f\| \leq K_{1} e^{-\gamma x}+K_{2} M \frac{1}{\gamma} .
$$


Let $\gamma>\gamma_{0}>K_{2}$, then for all $M \geq M_{0}=\frac{K_{1}}{1-\frac{K_{2}}{\gamma_{0}}}$ we have $K_{1} e^{-\gamma x}+K_{2} M \frac{1}{\gamma}<$ $M$. This is equivalent to saying that $S$ maps $G$ into itself, and therefore, the conclusion follows from Schauder's fixed point theorem. Now, for the case $n>3$, it is clear that all the computations that were performed for the $n=3$ case extend similarly, and the machinary of obtaining the existence remains true - the only difference is the size of the formulas. So we omit the details for the case $n>3$. This completes the proof of the theorem.

Remark 1. Note that in the definition of the subset $G$, the inequality $|f(g(x))|<|f(x)|$ has been implicitly invoked, and that the condition $g(x)<x$ on $I$ was instrumental to our method of proof. A better way to clarify things is perhaps to assume that $f(x)$ belongs to a class of functions $H$ defined as follows: Define a class of functions $D$ such that $f \in D$ if: (i) $f(x)$ is continous on $I$; and (ii) there exists a function $h(v)$ in $I$ such that $f(u v)<f(u) h(v)$. Note that (a) $D$ is a subspace of $E$; (b) the set of submultiplicative functions is a subset of $D$; and (c) if $p(x)$ and $r(x)$ are in $D$, then $p(x)+r(x), p(x) r(x)$, and $p(r(x))$ are in $D$. If further $h(v)=v$, we get a particular class which we call $H$. If $p(x)$ belongs to $H(I)$ and $\left(\frac{g(x)}{x}\right)<1$, then $p(g(x))=p\left(\frac{g(x)}{x} x\right)<$ $p(x) \frac{g(x)}{x}<p(x)$. In addition, for $t, s \in H$, we see that for $0 \leq \lambda \leq 1$, we have $\lambda t(u v)+(1-\lambda) s(u v) \leq(\lambda t(u)+(1-\lambda) s(u)) v$, and so as continuity is obvious, $H$ is convex. Furthermore, if we assume $H$ is a subset of the bounded continuous functions on $I$, that is, $\left\|h-h_{0}\right\| \leq \eta$, for some element $h_{0}$ and some constant $\eta$. Then it is straightforward to show that $H$ is complete. Take a convergent sequence $\left\{h_{n}\right\}$ with $h_{n}$ in $H,\left\{h_{n}\right\}$ converges to some element $k$ in $E$. Let $\varepsilon>0$. Then there is an $N$ such that if $n \geq N,\left\|h_{n}-k\right\|<\varepsilon$. Hence $\left\|k-h_{0}\right\| \leq\left\|k-h_{n}\right\|+\left\|h_{n}-h_{0}\right\|<\varepsilon+\eta$. As this is true for every $\varepsilon$, then $\left\|k-h_{0}\right\| \leq \eta$, and so $k$ is in $H$. Since $H$ is complete, it also follows that it is closed. Note now that if $f \in H, f(g(x))<f(x)$, and $g(x)<x$, and so we can conclude that $H$ is exchangable with $G$.

Remark 2. Note that in our proof of the uniqueness we did not assume but the continuity of the $a_{i}^{\prime} s$. So one can ask if it is possible to weaken the assumption in part (i) of Theorem 1 to assume only the continuity of the $a_{i}$ 's. To show that is possible, we employ the same method used above to derive (3). By the observation of Oberg mentioned above we may assume that $g(0)=0$. Carrying out the details, (1) can be shown to be equivalent to

$$
z(x)=a(x) \int_{0}^{g(x)} \frac{(g(x)-s)^{n-1}}{(n-1) !} z(s) d s
$$




$$
\begin{aligned}
& +b(x) \int_{0}^{x} \frac{(x-s)^{n-1}}{(n-1) !} z(s) d s-\sum_{i=1}^{n-1} a_{n-i}(x) \int_{0}^{x} \frac{(x-s)^{i-1}}{(i-1) !} z(s) d s \\
& +a(x) \sum_{k=1}^{n} \frac{g(x)^{n-k}}{(n-k) !} f^{(n-k)}(0)+b(x) \sum_{k=1}^{n} \frac{x^{n-k}}{(n-k) !} f^{(n-k)}(0) \\
& -\sum_{i=1}^{n-1} a_{n-i}(x)\left(\sum_{k=1}^{i} \frac{x^{i-k}}{(i-k) !} f^{(n-k)}(0)\right)+c(x) .
\end{aligned}
$$

Hence, we can also see, for $p$ in $H$ (and so $\frac{g(x)}{x}<1$ ), that

$$
\begin{aligned}
|(S p)(x)| & \leq \int_{0}^{x}\left[(A+B) \frac{(x-s)^{n-1}}{(n-1) !}+a \sum_{i=1}^{n-1} \frac{(x-s)^{i-1}}{(i-1) !}\right]|p(s)| e^{-\gamma s} e^{\gamma s} d s+F \\
& \leq K(n, L) \frac{e^{\gamma s}}{\gamma}\|p\|+F
\end{aligned}
$$

where $K(n, L), F$ are constants. So for large enough $\gamma$, we see that

$$
\|(S p)(\cdot)\| \leq \frac{K(n, L)}{\gamma}\|p\|+F e^{-\gamma L} \leq\|p\| .
$$

\section{References}

[1] T. Kato, J.B. McLeod, The functional differential equation $y^{\prime}(x)=$ ay $(\lambda x)+$ by $(x)$, Bull. Amer. Math. Soc., 77 (1971), 891-937.

[2] P.O. Frederickson, Dirichlet Series Solution for Certain Functional Differential Equations, Lecture Notes in Mathematics, 243, Springer-Verlag, New York (1971).

[3] Y.T. Siu, On solution of the equation $f^{\prime}(x)=\lambda f(g(x))$, Math. Zeit., 90 (1965), 391-392.

[4] G.R. Morris, A. Feldstein, E.W. Bowen, The Phragmén-Lindelöf principle and a class of functional differential equations, In: Ordinary Differential Equations, 1971, NRL-MRC Conference (Ed. L. Weiss), Academic Press, New York (1972), 513-540.

[5] J.R. Ockendon, A.B. Taylor, The dynamics of current collection system for an electric automotive, Proc. Royal Soc. of London, Series A, 322 (1971), 447-468. 
[6] G. Derfel, A. Iserles, The pantograph equation in the complex plane, $J$. Math. Anal. Appl., 213 (1997), 117-132.

[7] B. van Brunt, J.C. Marshall, G.C. Wake, Holomorphic solutions to pantograph type equations with neutral fixed points, J. Math. Anal. Appl., 295 (2004), 557-569.

[8] B.Q. Li, E.G. Saleeby, On solutions of the functional-differential equations $f^{\prime}(x)=a(x) f(g(x))+b(x) f(x)+c(x)$ in the large, Il. J. Math., 162 (2007), 335-348.

[9] D.R. Anderson, An existence theorem for a solution of $f^{\prime}(x)=$ $F(x, f(g(x)))$, SIAM Rev., 8 (1965), 98-105.

[10] R.J. Oberg, On the local existence of solutions of certain functionaldifferential equations, Proc. Amer. Math. Soc., 20 (1969), 259-302.

[11] R.J. Oberg, Local theory of complex functional differential equations, Trans. Amer. Math. Soc., 161 (1971), 269-281.

[12] R. Precup, Methods in Nonlinear Integral Equations, Kluwer, Netherlands (2002).

[13] F.G. Tricomi, Integral Equations, Dover, New York (1985). 